\title{
Improving Mesoscale Altimetric Data From a Multitracer Convolutional Processing of Standard Satellite-Derived Products
}

\author{
Fablet Ronan ${ }^{1,{ }^{*}}$, Verron Jacques ${ }^{2}$, Mourre Baptiste ${ }^{3}$, Chapron Bertrand ${ }^{4}$, Pascual Ananda 5
}

1 Inst Mines Telecom Atlantique, UMR LabSTICC 6285, FR-29238 Brest, France.

2 CNRS, Inst Geosci Environm, UMR 5183, Lab Glaciol \& Geophys Environm, FR-38058 Grenoble,

France.

${ }^{3}$ Sistema Observ \& Prediccio Costaner Illes Balears, Balearic IsI Coastal \& Forecasting Syst, E-07121

Palma De Mallorca, Spain.

4 IFREMER, Lab Oceanog Phys \& Spatiale, FR-29238 Brest, France.

${ }^{5}$ Mediterranean Inst Adv Studies CSIC UIB, E-07190 Esporles, Spain.

* Corresponding author: Ronan Fablet, email address : ronan.fablet@telecom-bretagne.eu

\begin{abstract}
:
Multisatellite measurements of altimeter-derived sea surface height (SSH) have provided a wealth of information on the ocean. Yet, horizontal scales below $100 \mathrm{~km}$ remain scarcely resolved. Especially, in the Mediterranean Sea, an important fraction of the mesoscale range, characterized by a small Rossby radius of deformation of $15-20 \mathrm{~km}$, is not properly retrieved by altimeter-derived gridded products. Here, we investigate a novel processing of AVISO products with a view to resolving the horizontal scales sensed by current along-track altimeter data. The key feature of our framework is the use of linear convolutional operators to model the fine-scale SSH detail as a function of different sea surface fields, especially optimally interpolated SSH and sea surface temperature (SST). The proposed model embeds the surface quasi-geostrophic SST-SSH synergy as a special case. Using an observing system simulation experiment with simulated SSH data from model outputs in the Western Mediterranean Sea, we show that the proposed approach has the potential for improving current optimal interpolations of gridded altimeterderived SSH fields by more than $20 \%$ in terms of relative SSH and kinetic energy mean square error, as well as in terms of spectral signatures for horizontal scales ranging from 30 to $100 \mathrm{~km}$. Our results also suggest that SST-SSH relationship may only play a secondary role compared with the interscale SSH cascade. We further discuss the relevance of the proposed approach in the context of future altimetric satellite missions.
\end{abstract}

Keywords : Convolutional models, observing system simulation experiment (OSSE), sea surface height (SSH), sea surface temperature (SST), superresolution, Western Mediterranean Sea 


\section{INTRODUCTION AND RELATED WORK}

In the last two decades, multi-satellite measurements of altimeter-derived Sea Surface Height (SSH) and multisensor measurements of Sea Surface Temperature (SST) have provided a wealth of information on ocean circulations $[2,5]$. As a depth-integrated quantity dependent upon the density structure of the water column, altimeter-derived SSH estimations capture mesoscale structures for horizontal scales of a few tens of kilometers to a few hundreds kilometers, and deliver an estimation of surface currents using the geostrophy balance. Yet, horizontal scales below $100 \mathrm{~km}$ remain scarcely resolved. Especially, in the Mediterranean Sea, an important fraction of mesoscale range, characterized by a small Rossby radius of deformation, $15-20 \mathrm{~km}$ a factor of 2-4 less than in the Global Ocean $[21,17]$. This scale range, which plays a major role both in the general ocean circulation through Eddy-Mean interactions as well as for ocean biogeochemistry and biology $[4,22]$, is not revealed by altimeter-derived L4 products, which rely on optimal interpolation of along-track altimeter data $[20,19]$. Note that this is even more true for the smaller scales know as submesoscales whose role on ocean dynamics and ocean biogeochemistry is more and more recognized and that might be accessed with the future altimetric sensors e.g. [27].

The needs to properly access the mesoscales, and more generally the oceanic small scales, have motivated a number of studies dedicated to the reconstruction of higher-resolution $\mathrm{SSH}$ fields using other strategies and multi-platform studies $[3,18]$. In this respect, several studies $[10,13,14]$ rationalize and demonstrate that SST fields can become an active tracer leading to strong correlations with $\mathrm{SSH}$ fields. In particular, SQG (Surface Quasi-Geostrophic) transfer functions and further linear extensions $[9,11,7,26]$ were investigated to predict high-resolution SSH fields from SST snapshots. Other studies have investigated other tracers, such as finitesize Lyapunov exponents [6] and local singularity exponents [24], to reconstruction the fine-scale details of the SSH fields, under the assumption that these tracer fields and the SSH field should share similar fine-scale geometrical patterns based on fine-scale eddy dynamics considerations. These studies mostly rely on an a priori assumption on the relationship between the unobserved high-resolution SSH and some observed sea surface tracers (e.g., SST, FSLE), whereas recent works show that upper ocean dynamics involve space-and-time-varying relationships $[11,15]$. Besides, they also mostly exploit the joint analysis of L4 gridded sea surface fields and do not explore the potential of along-track altimeter datasets, which embed relevant high-resolution along-track information.

In this study, we investigate the synergy between along-track altimeter data and L4 gridded sea surface fields with a view to reconstructing high-resolution SSH fields. Our contributions are two-fold:

- we first introduce a multi-tracer model to relate the highresolution SSH to observed sea surface tracers through convolutional operators along with the calibration of these operators from along-track altimeter data;

- Second, using an observing system simulation experiment (OSSE), we show for a case-study region in the western Mediterranean Sea that a convolution model combining 
optimal-interpolation-derived L4 $\mathrm{SSH}$ fields and highresolution SST fields can contribute to a significant improvement of the estimation of the $\mathrm{SSH}$ mesoscale variability for horizontal scales from $30 \mathrm{~km}$ to $100 \mathrm{~km}$, including spectral features.

This paper is organized as follows. Section II describes the proposed model for the improvement of mesoscale altimetric data. Section III presents the considered OSSE and the associated results. We further discuss our key contributions and future work in Section IV.

\section{PROPOSED APPROACH}

\section{A. Problem statement}

As stated in the introduction, our goal is to reconstruct highresolution SSH field from a joint analysis of L4 altimeterderived SSHs field, along-track altimeter datasets and highresolution SST fields. With a view to formally describing our model, we introduce the following notations:

- $S S H$ refers to the high-resolution SSH field to be reconstructed at a given reference date $t_{0}$. We drop the time index for all fields for the sake of simplicity;

- $\left\{t_{A T}(k), s_{A T}(k), S S H_{A T}(k)\right\}_{k}$ refers to a dataset of along-track data $\left\{S S H_{A T}(k)\right\}$ sampled by narrow-swath altimeters at times $\left\{t_{A T}(k)\right\}$ around reference date $t_{0}$ and positions $\left\{s_{A T}(k)\right\}$. The spatial sampling rate of the along-track data defines the lower bound of the considered fine-scale range ( $30 \mathrm{~km})$;

- $S S H_{L R}$ refers to a low-resolution reconstruction of the SSH field. It typically amounts to an optimal interpolation of along-track altimeter data $\left\{t_{A T}(k), s_{A T}(k), S S H_{A T}(k)\right\}_{k}[20]$;

- $S S T$ refers to a high-resolution SST field, typically a $1 \mathrm{~km}$-to-5km spatial resolution.

Here, we aim at reconstructing field $S S H$ given fields $S S H_{L R}$ and $S S T$ and along-track dataset $\left\{t_{A T}(k), s_{A T}(k), S S H_{A T}(k)\right\}_{k}$. Formally, we consider the following additive model:

$$
S S H=S S H_{L R}+H\left(S S H_{L R}, S S T\right)+N
$$

where $H$ is a transfer function, which states the contribution of the low-resolution SSH and the high-resolution SST to the reconstruction of the fine-scale component of the high-resolution SSH field. $N$ is regarded as a noise process accounting for unresolved scales. Hereafter, we refer to $S S H-S S H_{L R}$ as the high-resolution $\mathrm{SSH}$ detail, denoted by $d S S H$. Along-track dataset $\left\{t_{A T}(k), s_{A T}(k), S S H_{A T}(k)\right\}_{k}$ can be regarded as a set of observations of model (1).

\section{B. Convolution-based multi-tracer model}

In this study, we state model (1) according to linear convolution operators:

$$
S S H=S S H_{L R}+H_{1} * S S H_{L R}+H_{2} * S S T+N
$$

where $H_{1}$ (resp. $H_{2}$ ) states the contribution of the lowresolution $\mathrm{SSH}$ field (resp. the high-resolution SST field) to the reconstruction of the $\mathrm{SSH}$ detail. It may be noted that
SGQ dynamics [13, 9] may be regarded as a special case of (2), where the convolutional operators are parameterized as $H_{1}=\mathbf{0}$ and $H_{2}=\Delta^{-1 / 2}$ (a fractional Laplacian filter).

The above model relates to patch-based super-resolution image models [28, 8, 25]. It involves two main differences. First, it combines both a low-resolution observation and an additional high-resolution source (here, the SST field). Second, as detailed below, here we are provided for each data with a set of high-resolution sample points, through along-track altimeter data. No such data is available in the classical image superresolution and the model is trained offline from a dataset of high-resolution images. Given the expected time variability of operators $H_{1,2}$, we favor here local estimation robustness to model complexity.

Convolutional operators may be represented in the Fourier domain, as in the case of the fractional Laplacian operator associated with SQG dynamics [13,9]. Convolution operators may also be represented by their impulse response, which resorts to a convolution matrix when considering gridded data. This matricial representation in the spatial domain appears more adapted to deal with the sampling geometry of sea surface fields, especially regarding the presence of land areas (coastal areas, islands) and the irregular sampling of alongtrack altimeter data. Hence, operators $H_{1,2}$ are characterized by their spatial support $(2 * K+1) \times(2 * K+1)$. Here, for a grid resolution of $1 / 20^{\circ}$, parameter $\mathrm{K}$ typically range from 1 to 4 . Given parameter $K$, three types of parameterizations of convolution operators $H_{1,2}$ may be considered:

- "Full" models: operators $H_{1,2}$ are characterized by a $(2 *$ $K+1) \times(2 * K+1)$ matrix;

- "Isotropic" models: operators $H_{1,2}$ are characterized by a $(2 * K+1) \times(2 * K+1)$ matrix invariant to horizontal and vertical flipping and $90^{\circ}$ rotation. This isotropy constraint imposes the operators to depict the same filtering behaviour in all directions, what may be expected from a theoretical point of view;

- "Parametric" models: operators $H_{1,2}$ may be set according to predefined parametric forms. Here, we will test for operator $\mathrm{H}_{2}$ a SQG-related parameterization, that is to say $H_{2}=\alpha \Delta^{-1 / 2}$ with $\alpha$ a scalar parameter. We consider a $(2 * K+1) \times(2 * K+1)$ approximation of fractional Laplacian operator $\Delta^{-1 / 2}$, so that to the reconstruction error is minimized.

\section{Model calibration}

To implement reconstruction model (1), we first need to calibrate operators $H_{1,2}$. As stated previously, under the assumption that along-track samples $\left\{t_{A T}(k), s_{A T}(k), S S H_{A T}(k)\right\}_{k}$ are realizations of model (1), we resort to the minimization of the following least-square criterion

$$
\begin{array}{r}
\sum_{k} \| d S S H_{A T}(k)-H_{1} * S S H_{L R}\left(t_{A T}(k), s_{A T}(k)\right) \\
-H_{2} * S S T\left(t_{A T}(k), s_{A T}(k)\right) \|^{2}
\end{array}
$$

with $d S S H_{A T}(k)=S S H_{A T}(k)-S S H_{L R}\left(t_{A T}(k), s_{A T}(k)\right)$. It may be noted that along-track samples may not lie on the considered uniform grid, such that the convolution products 
$H_{1} * S S H_{L R} H_{2} * S S T$ at positions $\left\{t_{A T}(k), s_{A T}(k)\right\}$ require the interpolation of $(2 * K+1) \times(2 * K+1) S S H_{L R}$ and $S S T$ patches around each along-track positions $\left\{t_{A T}(k), s_{A T}(k)\right\}$. Given the matrical representation of operators $H_{1,2}$, the above minimization amounts to a linear regression. To avoid overfitting, the number of along-track samples should be significantly greater than the number of model parameters (i.e., $2 *(2 * K+1)^{2}$ for full models).

\section{Western Mediterranean Sea CASE-Study}

\section{A. Observing System Simulation Experiment (OSSE)}

To evaluate the proposed approach, we develop an OSSE from numerical simulations for a region in the western Mediterranean Sea from the South of Balearic islands to the Algerian coast $\left(36.5^{\circ} \mathrm{N}\right.$ to $40^{\circ} \mathrm{N}, 1.5^{\circ} \mathrm{E}$ to $\left.8.5^{\circ} \mathrm{N}\right)$. The considered OSSE involves three key components:

- Numerical simulations: we exploit the high-resolution numerical simulations of the WMOP model [12] from SOCIB (Balearic Islands Coastal Observing and Forecasting System, http://www.socib.eu/). We specifically use the daily SST and SSH fields from the free run hindcast simulation spanning the period 2009-2015. The model has a 2-km spatial resolution and is forced by 3-hourly atmospheric fields from the 5-km resolution HIRLAM model from the Spanish Meteorological Agency. Spatial averages over the specific reduced domains of study are removed from the daily SSH model outputs before our analysis in order to discard the large-scale signals including the high-frequency variability related to atmospheric pressure forcing and winds and so focus exclusively on the spatial scales below $300 \mathrm{~km}$.

- Simulations of along-track altimeter data: using periodization, we use the 2014 and 2015 series of real positions of along-track altimeter data from four altimeters to generate along-track positions for the entire time series of the simulations. We select year 2014 and 2015 as four altimeters were available, namely Saral/Altika, CryoSat2, HY-2 and OSTM/Jason-2. On a daily basis, we sample the simulated SSH field at generated along-track positions to extract pseudo along-track altimeter data. Prior to this sampling of the SSH fields, we apply a spatial filtering consistent with the along-track sampling resolution $(\tilde{1} 5 \mathrm{~km})$. The resulting along-track altimeter data refer to dataset $\left\{t_{A T}(k), s_{A T}(k), S S H_{A T}(k)\right\}_{k}$ introduced in Section II;

- Simulations of optimally-interpolated L4 SSH fields: from the simulated along-track dataset, we compute L4 SSH fields, referred to as low-resolution SSH field and denoted by $S S T_{L R}$, using an optimal interpolation with the following parameter setting. The spatial correlation length is set to $100 \mathrm{~km}$ and the temporal correlation length to 7 days. We consider Arhan covariance model [3] and a $1 / 8^{\circ}$ grid resolution. Those parameters are selected to mimic the operational L4 processing for gridded SSH fields [20].

An illustration of the simulated dataset are reported in Fig.2. Based on this OSSE, we apply the proposed approach which

\begin{tabular}{|l|l|l|l||}
\hline \hline & Relative & Relative & Relative \\
& SSH MSE & KE MSE & PSD MSE \\
\hline OI-derived SSH & 0.22 & 0.95 & 0.42 \\
$H_{1}+H_{2}$ & $\mathbf{0 . 1 7}$ & 0.63 & $\mathbf{0 . 3 1}$ \\
$H_{1}+H_{2} D T=\infty$ & 0.19 & 0.68 & 0.34 \\
$H_{1}+H_{2}=0$ & 0.19 & 0.68 & 0.31 \\
$H_{1}=0+H_{2}$ & 0.20 & 0.74 & 0.42 \\
$H_{1}+H_{2, S Q G}$ & 0.18 & 0.64 & 0.31 \\
$H_{1, I \text { so }}+H_{2, \text { Iso }}$ & 0.17 & $\mathbf{0 . 6 2}$ & $\mathbf{0 . 3 0}$ \\
\hline \hline
\end{tabular}

TABLE I: Reconstruction performance of different parameterizations of model (2) with respect to the optimally-interpolated SSH fields $(O I-S S H)$ : we report relative mean square error for the SSH field, the SSH gradient magnitude and the radiallyaverage power spectral density (PSD).

aims at reconstructing the high-resolution SSH field given an optimally-interpolated low-resolution version, along-track altimeter data and a simultaneous high-resolution cloud-free SST observation. Based on the OSSE, we can perform a quantitative evaluation in terms of relative mean square error (MSE) of the reconstructed SSH and of the associated kinetic energy, as well as in terms of spectral signatures for scales ranging from $30 \mathrm{~km}$ to $100 \mathrm{~km}$, for which we can expect some improvements given the along-track sampling resolution.

We proceed to a comparative evaluation of the reconstruction of SSH fields from model (2) with respect to the low-resolution optimally-interpolated SSH field. We consider different parameterizations for model (2), namely:

- $H_{1}+H_{2}$ refers to model (2) with full $3 \times 3$ convolutional models $(K=1)$. We consider \pm 10 days $\left(d T_{A T}=10\right)$ around each date to estimate operators $H_{1}$ and $H_{2}$, what amount approximatively to 1000 along-track positions for model fitting;

- $H_{2, \infty}+H_{2, \infty}$ is similar to $H_{1}+H_{2}$ except that parameter $d T_{A T}$ is set to $\infty$, such that the operators $H_{1}$ and $H_{2}$ are the mean operators trained from the entire time series.

- $H_{1}+H_{2}=0$ refers to model (2) where operator $\mathrm{H}_{2}$ is discarded, i.e. no prediction is derived from the SST field. Parameters $K$ and $d T_{A T}$ are the same as for parameterization $\mathrm{H}_{1}+\mathrm{H}_{2}$;

- $H_{1}=0+H_{2}$ refers to model (2) where operator $H_{2}$ is discarded, i.e. , i.e. no prediction is derived from the low-resolution SSH field. Parameters $K$ and $d T_{A T}$ are the same as for parameterization $\mathrm{H}_{1}+\mathrm{H}_{2}$;

- $H_{1}+H_{2, S Q G}$ refers to model (2) where operator $H_{2}$ is a discrete approximation of the fractional Laplacian operator associated with SQG dynamics [13, 9]. Parameters $K$ and $d T_{A T}$ are the same as for parameterization $H_{1}+H_{2}$;

- $H_{1, I s o}+H_{2, I s o}$ refers to model (2) where both operators $H_{1}$ and $H_{2}$ are restricted to isotropical operators. Parameters $K$ and $d T_{A T}$ are the same as for parameterization $H_{1}+H_{2}$.

We refer to the optimally-interpolated SSH as $O I-S S H$.

\section{B. Results}

We report in Tab.I the relative MSE (mean square error) (i.e.the MSE normalized with respect to the daily variance of 

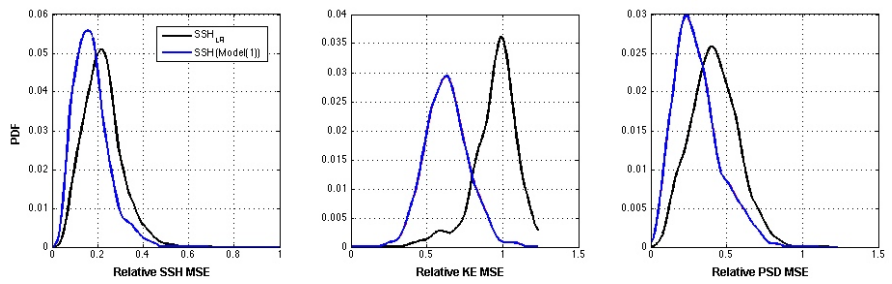

Fig. 1: Reconstruction statistics for the considered four-year time series: distribution of relative mean square error for SSH values (left), kinetic energy (center) and radially-averaged power spectral density (right). We compare the low-resolution optimally-interpolated field (black) to model (2) for parameterization $H_{1, \text { Iso }}+H_{2, \text { Iso }}$ (blue).

the high-resolution SSH field). All the considered parameterizations of model (2) lead to lower MSE values compared to $O I-S S H$ for all criterion (SSH, SSH gradient magnitude, PSD), though the gain is weak for parameterization $H_{1}=0+H_{2}$. Model $H_{1}+H_{2}$ leads to the lowest MSE values with a relative gain of $23 \%$ for the $\mathrm{SSH}, 34 \%$ for the SSH gradient and of $26 \%$ for the PSD. The distribution of the relative mse over the considered time series as depicted in Fig.1 further emphasizes the relevance of model (2) to go beyond the optimal interpolation for the reconstruction of high-resolution SSH variability below $100 \mathrm{~km}$.

The comparison of the different parameterizations of model (2) provides insights on the interpretation of model (2):

- The daily fit of operators $H_{1}$ and $H_{2}$ outperforms the mean operators $\left(d T_{A T}=\infty\right)(0.175$ vs. 0.186 in terms of relative SSH MSE). This points out the time variability of model (2), which may reveal the underlying variability of the upper ocean dynamics in the case-study area known to exhibit both seasonal and higher-frequency variabilities $[11,15,26]$.

- In model (2), operator $H_{1}$ associated to the low-resolution SSH field contributes the most to the prediction of the high-resolution SSH detail (relative SSH rmse of 0.186 for model $H_{1}+H_{2}=0$ w.r.t. 0.196 for model $H_{1}=$ $0+\mathrm{H}_{2}$ ). The anisotropical components of operator $H_{1}$ do not seem to be significant (relative SSH rmse of 0.175 for both model $H_{1}+H_{2}$ and $H_{1, \text { Iso }}+H_{2, \text { Iso }}$ );

- SST is not a negligible contributor in model (2) (relative SSH rmse of 0.175 for model $H_{1}+H_{2}$ including SST w.r.t. 0.186 for model $H_{1}+H_{2}=0$ without SST). Besides, the contribution from SST seems SQG-related as the SQG-based parameterization for operator $H_{2}$ reaches similar reconstruction performance than the full parameterization (relative SSH rmse of 0.175 for model $H_{1}+H_{2}$ w.r.t. 0.176 for model $H_{1}+H_{2, S Q G}=0$ ).

From Tab.I, we select parameterization $H_{1, \text { Iso }}+H_{2, \text { Iso }}$ in the subsequent analysis. We further illustrate the behavior of our approach from two contrasted examples, depicted in Fig.2 and Fig.3. The example during wintertime, February 10, 2010 , involves a strong correlation between the SST and SSH fields $\left(R^{2}=0.65\right)$. The relative SSH MSE is relatively large for the optimally-interpolated field (0.31). The reconstruction from model (2) enhances SSH gradients with finer SSH details, which leads to a lower relative MSE (0.22). This is emphasized by the visual inspection of the radially-averaged PSDs. The optimal interpolation clearly underestimates the energy below $100 \mathrm{~km}$, whereas the PSD associated with model (2) is more consistent with the true PSD. Consequently, model (2) resorts to a better estimation of the spectral slope in the range $[30 \mathrm{~km}, 100 \mathrm{~km}]$ ( 4.7 for the true SSH, 4.5 for the SSH reconstructed by model (2) and 5.7 for the optimallyinterpolated field). Similar observations can be drawn from the second example during summertime, February 10, 2010, with a lower correlation between SST and SSH $\left(R^{2}=0.35\right)$. Interestingly, these two examples exhibit different SST-SSH relationships. Whereas the sole use of SST in model (2), i.e. $H_{1}=0$, is relevant for the first example (relative SSH MSE of 0.22 w.r.t. 0.24 using $H_{1, \text { Iso }}+H_{2, \text { Iso }}$ parameterization), the SST field does not bring useful information in the second case to improve the reconstruction of the SSH field (relative SSH MSE of 0.07 for parameterzation $H_{1, I s o}+H_{2, \text { Iso }}$ and 0.09 for parameterization $H_{1, I s o}=0+H_{2, I s o}$ and the optimallyinterpolated SSH field).

As illustrated by the examples reported above, the proposed approach results in a better estimation of the spectral properties of the SSH field for horizontal scales ranging from $30 \mathrm{~km}$ to $100 \mathrm{~km}$. We depict in Fig. 4 the time series of the estimated spectral slopes for this scale range as well as their pdf. It clearly stresses the better match between the true SSH fields and the reconstruction using model (2) compared to the optimally-interpolated field. As such, model (2) provides a more consistent view of spectral properties of the upper ocean dynamics, including the expected link between SST and SSH spectral slopes under QG and SQG dynamical mode [? ].

\section{DISCUSSION}

We have presented and evaluated a novel model for the retreament of AVISO products to improve mesoscale altimetric data. Our model exploits convolutional operators and combines the information from both a low-resolution optimallyinterpolated SSH field (similar to AVISO L4-gridded product) and a high-resolution SST observation to reconstruct the SSH detail for horizontal scales not resolved by the optimal interpolation $(100 \mathrm{~km})$. Using an OSSE, we demonstrate the relevance of the proposed model to reconstruct the horizontal scales resolved by along-track data (up to $\tilde{3} 0 \mathrm{~km}$ ). We report significant improvements in terms of both SSH, SSH gradient reconstruction error as well as in terms of spectral signatures for a case-study area in the Western Mediterranean Sea. Interestingly, our study complements related works, who mainly investigated SQG-related priors [13, 9], and suggest that both low-resolution SSH and high-resolution SST convey relevant information to reconstruct upper ocean mesoscale dynamics, the former being on average the main contributor accounting for $75 \%$ of the relative gain in SSH MSE. In agreement with $[11,26]$, we exhibit time-varying operators. But, contrary to $[11,7]$, the anisotropical components of the convolution operators were shown to be negligible for the considered case-study and the SST contribution was mainly 

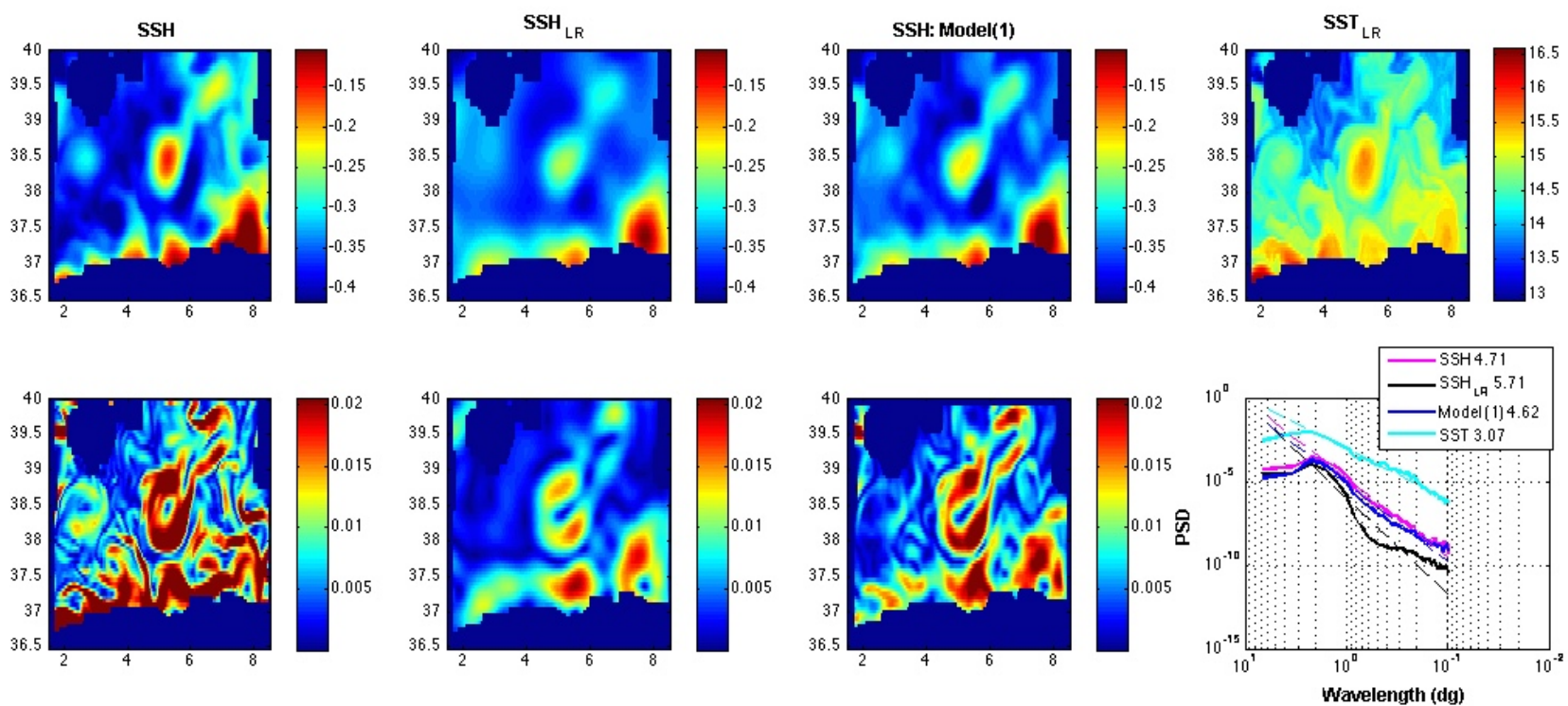

Fig. 2: High-resolution SSH reconstruction, February, 10, 2010: first row, from left to right, high-resolution SSH, low-resolution SSH, reconstructed SSH using model (2) with parameterization XXXX, high-resolution SST detail; second row, from left to right, gradient magnitudes of the high-resolution, low-resolution , and reconstructed SSH fields, radially-average power-spectral density of the high-resolution SSH (magenta), low-resolution SSH (black), reconstructed SSH (blue) and high-resolution SST (cyan). See the main for the detail on model parameterization.
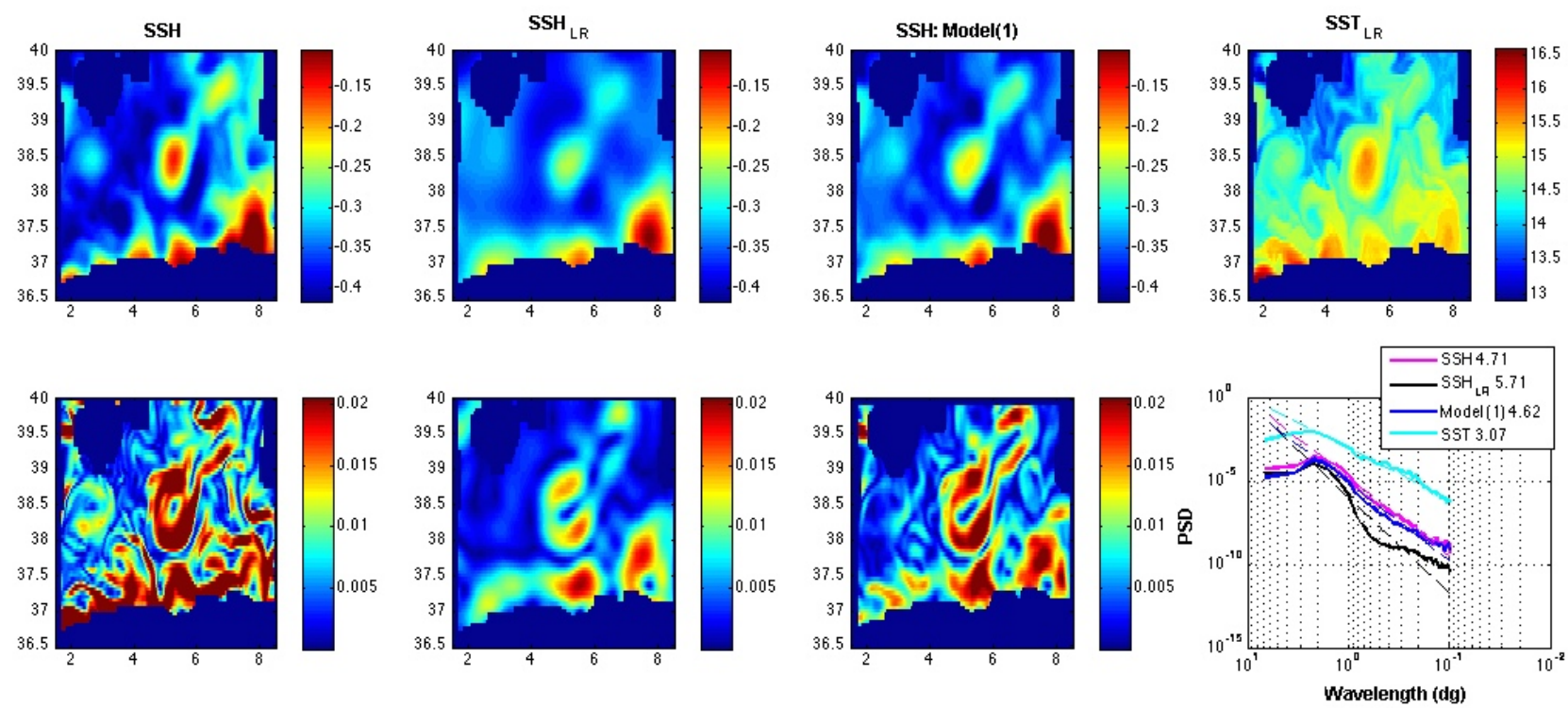

Fig. 3: High-resolution SSH reconstruction, August, 8, 2010: see Fig.2

SQG-related. This might be interpreted as a consequence of the combination of low-resolution SSH and high-resolution SST features in our model, whereas [11, 7] only considered SST-SSH relationships.

Besides the temporal variability of the considered convolutional operators, they are also expected to involve spatial variabilities, that will be of interest for future work and extension to regional and global scales. The proposed model makes also simple the potential combination of additional sea surface tracers, including for instance ocean colour [23] and Lagrangian signatures [6]. The extension to non-linear convolutional models $[16,1]$ seems a natural a extension to address the complexity of multi-tracer upper ocean dynamics, keeping in mind the balance model complexity and timevarying estimation robustness. This appears particularly appealing in the context of the future SWOT mission which will provide more spatially-structured data for model calibration [].

\section{ACKNOWLEDGMENT}

This work was supported by ANR (Agence Nationale de la Recherche, grant ANR-13-MONU-0014), Labex Cominlabs (grant SEACS) and visiting grant from UIB (University of 

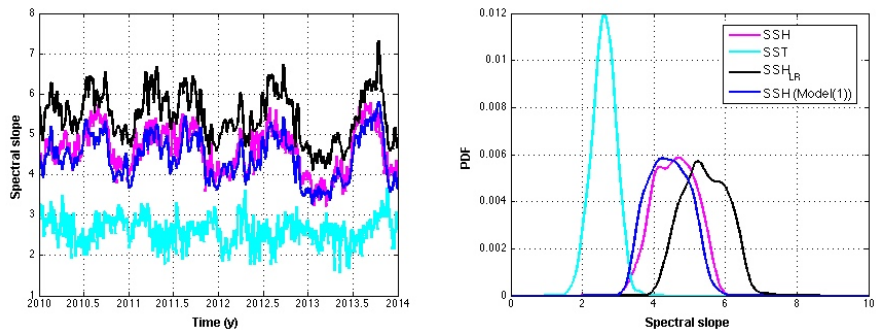

Fig. 4: Estimation of spectral slopes for the considered fouryear time series: time series of the estimated spectral slopes (left), distribution of the estimated spectral slopes (right). We depict the spectral slopes of the radially-averaged power spectral density of the high-resolution SSH (magenta), the lowresolution SSH (black), the reconstructed SSH (blue) and the high-resolution SST (cyan).

Balearic Islands). AP was supported by the Copernicus Marine Environment Monitoring Service (CMEMS) through the Sea Level Thematic Assembly Center (SLTAC).

\section{REFERENCES}

[1] C. Dong, Chen Change Loy, K. He, and X. Tang. Learning a Deep Convolutional Network for Image SuperResolution. In D. Fleet, T. Pajdla, B. Schiele, and T. Tuytelaars, editors, Computer Vision ECCV 2014: 13th European Conference, Zurich, Switzerland, September 6-12, 2014, Proceedings, Part IV, pages 184-199. Springer International Publishing, Cham, 2014.

[2] C. Donlon. The Next Generation of Multi-Sensor Merged Sea Surface Temperature Data Sets for Europe. In Vittorio Barale and Martin Gade, editors, Remote Sensing of the European Seas, pages 177-188. Springer Netherlands, 2008. DOI: 10.1007/978-1-4020-6772-3_14.

[3] R. Escudier, J. Bouffard, A. Pascual, P.-M. Poulain, and M.-I. Pujol. Improvement of coastal and mesoscale observation from space: Application to the northwestern Mediterranean Sea. Geophysical Research Letters, 40(10):2148-2153, 2013.

[4] L.-L. Fu, D. Allemand, P.-Y. Le Traon, and R. Morrow. Eddy Dynamics From Satellite Altimetry. Oceanography, 23(4):14-25, December 2010.

[5] L.-L. Fu and A. Cazenave. Satellite Altimetry and Earth Sciences - Elsevier. Elsevier, 2001.

[6] L. Gaultier, Jacques Verron, Jean-Michel Brankart, Olivier Titaud, and Pierre Brasseur. On the inversion of submesoscale tracer fields to estimate the surface ocean circulation. Journal of Marine Systems, 126:3342, October 2013.

[7] C. Gonzlez-Haro and J. Isern-Fontanet. Global ocean current reconstruction from altimetric and microwave SST measurements. Journal of Geophysical Research: Oceans, 119(6):3378-3391, June 2014.

[8] H. He and W. C. Siu. Single image super-resolution using Gaussian process regression. In 2011 IEEE Conference on Computer Vision and Pattern Recognition (CVPR), pages 449-456, June 2011.
[9] J. Isern-Fontanet, B. Chapron, G. Lapeyre, and P. Klein. Potential use of microwave sea surface temperatures for the estimation of ocean currents. GEOPHYSICAL RESEARCH LETTERS, 33, 2006. L24608.

[10] J. Isern-Fontanet, G. Lapeyre, P. Bach, B. Chapron, and M. W. Hecht. Three-dimensional reconstruction of oceanic mesoscale currents from surface information. Journal of Geophysical Research: Oceans, 113(C9):C09005, September 2008.

[11] J. Isern-Fontanet, M. Shinde, and C. Andersson. On the Transfer Function between Surface Fields and the Geostrophic Stream Function in the Mediterranean Sea. Journal of Physical Oceanography, 44(5):1406-1423, March 2014.

[12] M. Juza, B. Mourre, L. Renault, S. Gmara, K. Sebastin, S. Lora, J. P. Beltran, B. Frontera, B. Garau, C. Troupin, M. Torner, E. Heslop, B. Casas, R. Escudier, G. Vizoso, and J. Tintor. SOCIB operational ocean forecasting system and multi-platform validation in the Western Mediterranean Sea. Journal of Operational Oceanography, 9(sup1):s155-s166, February 2016.

[13] P. Klein, J. Isern-Fontanet, G. Lapeyre, G. Roullet, E. Danioux, B. Chapron, S. Le Gentil, and H. Sasaki. Diagnosis of vertical velocities in the upper ocean from high resolution sea surface height. Geophysical Research Letters, 36(12):L12603, June 2009.

[14] J. H. LaCasce and A. Mahadevan. Estimating subsurface horizontal and vertical velocities from sea-surface temperature. Journal of Marine Research, 64(5):695-721, September 2006.

[15] C. Le Goff, R. Fablet, P. Tandeo, E. Autret, and B. Chapron. Spatio-temporal decomposition of satellitederived SST-SSH fields: links between surface data and ocean interior dynamics in the Agulhas region. IEEE Journal of Selected Topics in Applied Earth Observations and Remote Sensing, 2016.

[16] Y. LeCun, Y. Bengio, and G. Hinton. Deep learning. Nature, 521(7553):436-444, 2015.

[17] P. Malanotte-Rizzoli, V. Artale, G. L. Borzelli-Eusebi, S. Brenner, A. Crise, M. Gacic, N. Kress, S. Marullo, M. Ribera d'Alcal, S. Sofianos, T. Tanhua, A. Theocharis, M. Alvarez, Y. Ashkenazy, A. Bergamasco, V. Cardin, S. Carniel, G. Civitarese, F. D'Ortenzio, J. Font, E. Garcia-Ladona, J. M. Garcia-Lafuente, A. Gogou, M. Gregoire, D. Hainbucher, H. Kontoyannis, V. Kovacevic, E. Kraskapoulou, G. Kroskos, A. Incarbona, M. G. Mazzocchi, M. Orlic, E. Ozsoy, A. Pascual, P.-M. Poulain, W. Roether, A. Rubino, K. Schroeder, J. Siokou-Frangou, E. Souvermezoglou, M. Sprovieri, J. Tintor, and G. Triantafyllou. Physical forcing and physical/biochemical variability of the Mediterranean Sea: a review of unresolved issues and directions for future research. Ocean Sci., 10(3):281-322, May 2014.

[18] A. Pascual, J. Bouffard, S. Ruiz, B. Buongiorno Nardelli, E. Vidal-Vijande, R. Escudier, J. M. Sayol, and A. Orfila. Recent improvements in mesoscale characterization of the western Mediterranean Sea: synergy between satellite altimetry and other observational approaches. Scientia 
Marina, 77(1):19-36, March 2013.

[19] A. Pascual, M.-I. Pujol, G. Larnicol, P.-Y. Le Traon, and M.-H. Rio. Mesoscale mapping capabilities of multisatellite altimeter missions: First results with real data in the Mediterranean Sea. Journal of Marine Systems, 65(14):190-211, March 2007.

[20] M. I. Pujol and G. Larnicol. Mediterranean sea eddy kinetic energy variability from 11 years of altimetric data. Journal of Marine Systems, 58(34):121-142, December 2005.

[21] A. R. Robinson, W. G. Leslie, A. Theocharis, and A. Lascaratos. Mediterranean Sea Circulation A2 - Steele, John H. In Encyclopedia of Ocean Sciences, pages 1689-1705. Academic Press, Oxford, 2001.

[22] J. Rodrguez, J. Tintor, John T. Allen, J.M. Blanco, D. Gomis, A. Reul, J. Ruiz, V. Rodrguez, F. Echevarra, and F. Jimnez-Gmez. Mesoscale vertical motion and the size structure of phytoplankton in the ocean. Nature, 410(6826):360-363, March 2001.

[23] M. Saraceno, C. Provost, and A. R. Piola. On the relationship between satellite-retrieved surface temperature fronts and chlorophyll a in the western South Atlantic. Journal of Geophysical Research: Oceans, 110(C11):C11016, November 2005.

[24] J. Sudre, H. Yahia, O. Pont, and V. Garon. Ocean Turbulent Dynamics at Superresolution From Optimal Multiresolution Analysis and Multiplicative Cascade. IEEE Transactions on Geoscience and Remote Sensing, 53(11):6274-6285, November 2015.

[25] R. Szeliski. Computer Vision: Algorithms and Applications. Springer edition, 2010.

[26] P. Tandeo, B. Chapron, S. Ba, E. Autret, and R. Fablet. Segmentation of Mesoscale Ocean Surface Dynamics Using Satellite SST and SSH Observations. IEEE Transactions on Geoscience and Remote Sensing, 52(7):42274235, July 2014.

[27] Leif N. Thomas, Amit Tandon, and Amala Mahadevan. Submesoscale Processes and Dynamics. In thew W. Hecht and Hiroyasu Hasumi, editors, Ocean Modeling in an Eddying Regime, pages 17-38. American Geophysical Union, 2008.

[28] Chih-Yuan Yang, Chao Ma, and Ming-Hsuan Yang. Single-Image Super-Resolution: A Benchmark. In David Fleet, Tomas Pajdla, Bernt Schiele, and Tinne Tuytelaars, editors, Computer Vision ECCV 2014: 13th European Conference, Zurich, Switzerland, September 6-12, 2014, Proceedings, Part IV, pages 372-386. Springer International Publishing, Cham, 2014. 